\title{
Some Properties of Second Order Differential Equations
}

\author{
Qusuay H. AlqIFiary
}

\begin{abstract}
In this paper we established the Hyers-Ulam-Rassias stability of a linear differential equation of second order with initial condition. We also proved the Hyers-Ulam-Rassias stability of a nonlinear differential equation of second order with initial condition.
\end{abstract}

\section{INTRODUCTION}

In 1940, Hyers-Ulam stability was introduced by S.M. Ulam [7] to raise the question: suppose one has a function $y(t)$ which is close to solve an equation. Is there an exact solution $x(t)$ of the equation which is close to $y(t)$ ? (See [1, 12]). In 1941, D.H. Hyers [1] gave an affirmative answer to the equation of Ulam for additive Cauchy equation in Banach spaces. A generalized solution to Ulam's problem for approximately linear mappings was proved by Th.M. Rassias in 1978. Th. M. Rassias [11] considered a mapping $f: E_{1} \rightarrow E_{2}$ such that $t \mapsto f(t x)$ is continuous in $t$ for each fixed $x$. Assume that there exists $\theta \geq 0$ and $0 \leq p<1$ such that

$$
\|f(x+y)-f(x)-f(y)\| \leq \theta\left(\|x\|^{p}+\|y\|^{p}\right)
$$

for any $x, y \in E_{1}$.

After that, many mathematicians have extended Ulam's problem in various directions (see $[2,6,13]$ ). A Hyers-Ulam-Rassias problem with differential equations:

The differential equation $\phi\left(f, y, y^{\prime}, \ldots, y^{(n)}\right)=0$ has the Hyers-Ulam-Rassias stability with respect to function $\theta$ if there exist a constant $M>0$ such that for given $\varepsilon>0$ and a function $y$ such that $\left|\phi\left(f, y, y^{\prime}, \ldots, y^{(n)}\right)\right| \leq \varepsilon \theta(t)$, there exists a solution $y_{c}$ of the differential equation such that $\left|y(t)-y_{c}(t)\right| \leq$ $M \varepsilon \theta(t)$.

Definition of Hyers-Ulam-Rassias stability have applicable significance since it means that if one is studying an Hyers-Ulam-Rassias stable system then one does not have to reach the exact solution.(which usually is quite

2010 Mathematics Subject Classification. Primary: 34-02; Secondary: 34K20, 26D10.

Key words and phrases. Differential equations, Hyers-Ulam-Rassias stability, Linear, Nonlinear, Second order. 
difficult or time consuming). This is quite useful in many applications e.g. numerical analysis, optimization,biology and economics etc., where finding the exact solution is quite difficult.

Jung has proved the Hyers-Ulam stability of linear differential equations (see $[8,10,9])$.

In this paper we will study the Hyers-Ulam-Rassias stability of a linear and nonlinear differential equations of second order. There are two main sections: in section(2), we will prove the Hyers-Ulam-Rassias stability of the linear differential equation of second order

$$
z^{\prime \prime}+p(t) z^{\prime}+(q(t)-\alpha(t)) z=0
$$

with initial conditions

$$
z\left(t_{0}\right)=0=z^{\prime}\left(t_{0}\right)
$$

where $q \in C^{0}(I), p \in C^{1}(I), I=\left[t_{0}, t\right] \subseteq \mathbb{R}, t_{0}>0, P(t)>0$ and $\alpha(t)$ is a bounded for all sufficiently large $t$ in $R$. In addition to that in Section 3 we investigate the Hyers-Ulam-Rassias stability of the following nonlinear differential equation of second order

$$
z^{\prime \prime}+p(t) z^{\prime}+q(t) z=h(t)|z|^{\beta} e^{\left(\frac{\beta-1}{2}\right) \int p(t) \mathrm{d} t} \operatorname{sgn} z, \quad \beta \in(0,1)
$$

with the initial conditions

$$
z\left(t_{0}\right)=0=z^{\prime}\left(t_{0}\right)
$$

where $h \in C^{1}(I)$ and $h(t)$ is a bounded for all sufficiently large $t$ in $R$. It should note here that we may assume that $z>0$ in (3) because if $z<0$ we set $z=-u, u>0$. So we will consider in future the equation

$$
z^{\prime \prime}+p(t) z^{\prime}+q(t) z=h(t) z^{\beta} e^{\left(\frac{\beta-1}{2}\right) \int p(t) \mathrm{d} t}, \quad \beta \in(0,1) .
$$

\section{Hyers-Ulam-Rassias stability of the linear differential EQUATION OF SECOND ORDER}

\subsection{Preliminaries.}

Definition 1. We will say that (1) has the Hyers-Ulam-Rassias stability with $\theta(t)$, where $\theta: \mathbb{R} \rightarrow[0, \infty)$ and the initial conditions (2) if there exists a positive constant $K>0$ with the following property:

For every $\epsilon>0, z \in C^{2}(I)$ where $\mathrm{t}$ is sufficiently large in $\mathbb{R}$, if

$$
\left|z^{\prime \prime}+p(t) z^{\prime}+(q(t)-\alpha(t)) z\right| \leq \epsilon \theta(t)
$$

then there exists some solution $w \in C^{2}(I)$ of (1), such that $|z(t)-w(t)| \leq$ $K \epsilon \theta(t)$ and satisfies the initial conditions

$$
w\left(t_{0}\right)=0=w^{\prime}\left(t_{0}\right) .
$$


Lemma 1 (see [5]). A substitution $z(t)=y(t) e^{-\frac{1}{2} \int p(t) \mathrm{d} t}$ reduces $(1)$ to $(8)$

$$
y^{\prime \prime}+y=\alpha(t) y
$$

where

$$
q(t)-\frac{1}{4} p^{2}(t)-\frac{1}{2} p^{\prime}(t)=1
$$

Lemma 2 (see [4]). Suppose that $|\alpha(t)| \leq L$ for all $t \geq t_{0}$. If $L<1$ then any solution of $(8)$ is bounded as $t \rightarrow \infty$.

\subsection{Main Results I.}

Theorem 1. Suppose that $|\alpha(t)| \leq L<1$ for all $t \geq t_{0}$, and $y \in C^{2}(I)$, such that $\left|y^{\prime}(t)\right| \leq|y(t)|$ and satisfies the inequality

$$
\left|y^{\prime \prime}+y-\alpha(t) y\right| \leq \epsilon \theta(t)
$$

with the initial condition

$$
y\left(t_{0}\right)=0=y^{\prime}\left(t_{0}\right) .
$$

Then (8) with initial condition (11) has the Hyers-Ulam-Rassias stability with respect to $\theta(t)$ such that $\theta\left(t_{0}\right)=0$.

Proof. Suppose that $\epsilon>0$ and $y \in C^{2}(I)$, such that $\left|y^{\prime}(t)\right| \leq|y(t)|$ and satisfies (10) with the initial conditions (11) and $M=\max _{t \geq t_{0}}|y(t)|$.

From the inequality (10) we have

$$
-\epsilon \theta(t) \leq y^{\prime \prime}+y-\alpha(t) y \leq \epsilon \theta(t) .
$$

Multiply the inequality (12) by $y^{\prime}$ and then integrate we obtain

$$
\begin{aligned}
-2 \epsilon \int_{t_{0}}^{t} y^{\prime}(x) \theta(x) \mathrm{d} x & \leq y^{\prime 2}(t)+y^{2}(t)-2 \int_{t_{0}}^{t} \alpha(x) y(x) y^{\prime}(x) \mathrm{d} x \\
& \leq 2 \epsilon \int_{t_{0}}^{t} y^{\prime}(x) \theta(x) \mathrm{d} x .
\end{aligned}
$$

From which we get that

$$
\begin{aligned}
y^{2}(t) & \leq 2 \int_{t_{0}}^{t} \alpha(x) y(x) y^{\prime}(x)+2 \epsilon \int_{t_{0}}^{t} y^{\prime}(x) \theta(x) \\
& =2 \epsilon \int_{t_{0}}^{t} y^{\prime}(x) \theta(x)+\alpha\left(t^{*}\right) y^{2}(t) \\
& \leq 2 \epsilon M \theta(t)+L M^{2}
\end{aligned}
$$


Therefore

$$
M \leq \frac{2 \epsilon}{1-L} \theta(t) .
$$

Hence $|y(t)| \leq K \epsilon \theta(t)$, for all $t \geq t_{0}$. Obviously, $w(t)=0$ satisfies (8) and the zero initial conditions (11) such that

$$
|y(t)-w(t)| \leq K \epsilon \theta(t) .
$$

Hence (8) with initial condition (11) has the Hyers-Ulam-Rassias stability.

Corollary 1. Suppose that $|\alpha(t)| \leq L<1$ for all $t \geq t_{0}$, and $z \in C^{2}(I)$, such that $\left|z^{\prime}(t)\right| \leq|z(t)|$ and satisfies the inequality (6) with the initial condition (2). If the integral $\int_{t_{0}}^{\infty} p(t) \mathrm{d} t$ converges then (1) has the Hyers-Ulam-Rassias stability with initial condition (2).

Proof. Suppose that $z \in C^{2}(I)$, such that $\left|z^{\prime}(t)\right| \leq|z(t)|$ and satisfies the inequality (6). From the Theorem 1 it follows that (8) has the Hyers-UlamRassias stability with initial condition (11) and according to the substitution in Lemma 1 it follows that (1) has the Hyers-Ulam-Rassias stability with initial condition (2).

3. Hyers-Ulam-RAssias Stability of the nONLINEAR DifFERENTial EQUATION OF SECOND ORDER

\subsection{Preliminaries.}

Definition 2. We will say that (5) has the Hyers-Ulam-Rassias stability with $\theta(t)$, where $\theta: \mathbb{R} \rightarrow[0, \infty)$ and the initial conditions (4) if there exists a positive constant $K>0$ with the following property:

For every $\epsilon>0, z \in C^{2}(I)$ where $t$ is sufficiently large in $\mathbb{R}$, if

$$
\left|z^{\prime \prime}+p(t) z^{\prime}+q(t) z-h(t) z^{\beta} e^{\left(\frac{\beta-1}{2}\right) \int p(t) \mathrm{d} t}\right| \leq \epsilon \theta(t)
$$

then there exists some solution $w \in C^{2}(I)$ of (5), such that $|z(t)-w(t)| \leq$ $K \epsilon \theta(t)$ and satisfies the initial conditions

$$
w\left(t_{0}\right)=0=w^{\prime}\left(t_{0}\right) .
$$

Lemma 3 (see [5]). A substitution $z(t)=y(t) e^{-\frac{1}{2} \int p(t) \mathrm{d} t}$ reduces (5) to (16).

$$
y^{\prime \prime}+y=h(t) y^{\beta}, \quad \beta \in(-1,0) \cup(0,1)
$$

where

$$
q(t)-\frac{1}{4} p^{2}(t)-\frac{1}{2} p^{\prime}(t)=1 .
$$

Lemma 4 (see [5]). Suppose that $h(t)$ is a continuously differentiable function, bounded for all sufficiently large $t \in R$, and that the integral $\int_{t_{0}}^{\infty}\left|h^{\prime}(t)\right| \mathrm{d} t$ is convergent then any solution of (16) is bounded as $x \rightarrow \infty$. 


\subsection{Main Results II.}

Theorem 2. Suppose $|h(t)| \leq A$ for all $t \geq t_{0}$, and $y \in C^{2}(I)$, such that $\left|y^{\prime}(t)\right| \leq|y(t)|$ and satisfies the inequality

$$
\left|y^{\prime \prime}+y-h(t) y^{\beta}\right| \leq \epsilon \theta(t), \quad \beta \in(0,1)
$$

with initial condition

$$
y\left(t_{0}\right)=0=y^{\prime}\left(t_{0}\right)
$$

If $A \leq \frac{(\beta+1)}{2}\left(\max _{t \geq t_{0}}|y(t)|\right)^{-\beta}$, for $t \geq t_{0}$, then the equation

$$
y^{\prime \prime}+y=h(t) y^{\beta}, \quad \beta \in(0,1)
$$

has the Hyers-Ulam-Rassias stability with initial condition (19).

Proof. Suppose that $\epsilon>0$ and $y \in C^{2}(I)$, such that $\left|y^{\prime}(t)\right| \leq|y(t)|$ and satisfies (18) with the initial conditions (19) and $M=\max _{t \geq t_{0}}|y(t)|$.

From the inequality (18) we have

$$
-\epsilon \theta(t) \leq y^{\prime \prime}+y-h(t) y^{\beta} \leq \epsilon \theta(t) .
$$

Multiply the inequality (21) by $y^{\prime}$ and integrate we obtain

$$
\begin{aligned}
-2 \epsilon \int_{t_{0}}^{t} y^{\prime}(x) \theta(x) \mathrm{d} x & \leq y^{2}(t)+y^{2}(t)-2 \int_{t_{0}}^{t} h(x) y^{\prime}(x) y^{\beta}(x)^{\prime} \mathrm{d} x \\
& \leq 2 \epsilon \int_{t_{0}}^{t} y^{\prime}(x) \theta(x) \mathrm{d} x .
\end{aligned}
$$

From which we get that

$$
\begin{aligned}
y^{2}(t) & \leq 2 \int_{t_{0}}^{t} h(x) y^{\prime}(x) y^{\beta}(x) \mathrm{d} x+2 \epsilon \int_{t_{0}}^{t} y^{\prime}(x) \theta(x) \mathrm{d} x \\
& =\frac{2 h\left(t^{*}\right) y^{\beta+1}}{\beta+1}+2 \epsilon M \theta(t) \leq \frac{2 A M^{\beta+1}}{\beta+1}+2 \epsilon M \theta(t) .
\end{aligned}
$$

Therefore

$$
M \leq \frac{2 \epsilon}{1-\frac{2 A M^{\beta-1}}{\beta+1}} \theta(t) .
$$

Hence $|y(t)| \leq K \epsilon \theta(t)$, for all $t \geq t_{0}$. Obviously, $w(t)=0$ satisfies (20) and the zero initial conditions (19) such that

$$
|y(t)-w(t)| \leq K \epsilon \theta(t) .
$$

Hence (20) with initial condition (19) has the Hyers-Ulam-Rassias stability. 
Corollary 2. Assume that $h(x)$ and $z(t)$ satisfy the conditions of Theorem 2, and the inequality (14) with initial condition (4).

If $A \leq \frac{(\beta+1)}{2}\left(\max _{t \geq t_{0}}|y(t)|\right)^{-\beta}$, for $t \geq t_{0}$ and the integral $\int_{t_{0}}^{\infty} p(t) \mathrm{d} t$ converges then (5) has the Hyers-Ulam-Rassias stability with initial condition (2).

Proof. Suppose that $z \in C^{2}(I)$, such that $\left|z^{\prime}(t)\right| \leq|z(t)|$ and satisfies the inequality (14) with initial condition (4). From the Theorem 2 it follows that (20) has the Hyers-Ulam-Rassias stability with initial condition (19) and according to the substitution in Lemma 3 it follows that (5) has the Hyers-Ulam-Rassias stability with initial condition (4).

\section{REFERENCES}

[1] D.H. Hyers, On the stability of the linear functional equation, Proc. Natl. Soc. USA 27 (1941), 222-224.

[2] D. Popa, Hyers-Ulam-Rassias stability of a linear recurrence, J. Math. Anal. Appl. 309 (2005), 591-597.

[3] H. Rezaei, S.-M. Jung and Th. M. Rassias, Laplace transform and Hyers-Ulam stability of linear differential equations, J. Math. Anal. Appl. 403 (2013), 244-251.

[4] M.N. Qarawani, Hyers-Ulam stability of linear and nonlinear differential equations of second order, I.J. ofApplied Mathematics Research 1(4) (2012), 422-432.

[5] M.N. Qarawani, Boundedness and asymptotic behaviour of solutions of a second order nonlinear differential equation, J. of Mathematics Research 4(3) (2012), 121-128.

[6] P. Găvrută and L. Găvrută, A new method for the generalized Hyers-Ulam-Rassias stability, Internat. J. Nonlinear Anal. 2 (2010), 11-18.

[7] S.M. Ulam, Problems in Modern Mathematics, Chapter VI, Scince Editors, Wiley, New York, 1960.

[8] S.-M. Jung, Hyers-Ulam stability of linear differential equations of first order, Appl. Math. Lett. 17 (2004), 1135-1140.

[9] S.-M. Jung, Hyers-Ulam stability of linear differential equations of first order, III, J. Math. Anal. Appl. 311 (2005), 139-146.

[10] S.-M. Jung, Hyers-Ulam stability of linear differential equations of first order, II, Appl. Math. Lett. 19 (2006), 854-858.

[11] Th.M. Rassias, On the stability of the linear mapping in Banach spaces, Proc. Amer. Math. Soc. 72 (1978), 297-300.

[12] Y. Li, Y. Shen, Hyers-Ulam stability of nonhomogeneous linear differential equations of second order, Internat. J. Math. Math. Sci. 2009 (2009), Article ID 576852, 7 p.

[13] Y.-H. Lee and K.-W. Jun, A generalization of Hyers-Ulam-Rassias stability of Jensen's equation, J. Math. Anal. Appl. 238 (1999), 305-315.

Qusuay H. Alqifiary

University of Belgrade

BELgRAde

SERBia

Work: The University of Al-QAdisiyah

Al-DiWANiYA

IRAQ

E-mail address: qhaq2010@gmail.com 